\section{$\underset{\substack{\text { hommes } \\ \text { \& migrations }}}{ }$}

\section{Hommes \& migrations}

Revue française de référence sur les dynamiques

migratoires

1317-1318 | 2017

L'Europe en mouvement

\title{
« Expatriates in France », toujours plus d'Europe
}

\section{Catherine Ferioli Gavenc}

\section{CpenEdition \\ Journals}

\section{Édition électronique}

URL : http://journals.openedition.org/hommesmigrations/3916

DOI : 10.4000/hommesmigrations.3916

ISSN : 2262-3353

\section{Éditeur}

Musée national de l'histoire de l'immigration

\section{Édition imprimée}

Date de publication : 1 avril 2017

Pagination : 154-157

ISBN : 978-2-919040-38-4

ISSN : $1142-852 X$

\section{Référence électronique}

Catherine Ferioli Gavenc, « «Expatriates in France », toujours plus d'Europe », Hommes \& migrations [En ligne], 1317-1318 | 2017, mis en ligne le 01 avril 2017, consulté le 15 septembre 2020. URL : http:// journals.openedition.org/hommesmigrations/3916 


\title{
«XPATRIATES IN FRANCE », TOUJOURS PLUS D'EUROPE
}

\author{
Par CATHERINE FERIOLI GAVENC, directrice de la communication \\ et des partenariats, Responsable Éducation nationale, Casden.
}

$\mathrm{D}$ epuis plus de 25 ans, la Société de banque et d'expansion (SBE) est partenaire de grandes entreprises, industrielles, tertiaires dans des secteurs divers (recherche et développement, site de production ou de siège d'entreprise). Elle rencontre de nombreux interlocuteurs au profil différents (directeurs de ressources humaines, directeurs de site ou de sociétés de relocation) et s'est adaptée à leurs besoins et à la culture de leur entreprise. Quelle que soit l'organisation, sa préoccupation est d'apporter un suivi et un accompagnement à la mobilité nationale ou internationale, notamment en direction du personnel étranger installé en France. La SBE est donc un acteur bancaire de premier plan pour les » impatrié "s, ceux notamment qui prennent de nouvelles fonctions au sein du siège ou filiale de leur Groupe en France. Elle s'est associée à www. paris21.tv, web TV traduite en 7 langues, dédiée aux étrangers résidant en France qui appartient à la société Mondissimo, laquelle est spécialisée depuis 25 ans dans la communication auprès des expatriés.

Pour rappel, le mot «Impatrié » se définit en opposition au mot expatrié. C'est un salarié ou dirigeant appelé à occuper un emploi pendant une durée déterminée ou indéterminée dans une entreprise établie en France, soit par l'intermé- diaire d'une entreprise établie à l'étranger, détenant des filiales en France ou bien directement par l'entreprise établie en France, un groupe français détenant des filiales à l'étranger. Ce peut être encore un travailleur indépendant ou exerçant une profession libérale. En 2014 la première étude a été lancée sous forme d'enquête, intitulée "Expatriates in France: Tell us about your life... " qui présentait différents aspects la vie de ces résidents en France (note sur article dans $H \& M)$.

2016, réalisée sur site internet par bannière de recrutement, a recueilli les réponses de plus de 1248 impatriés. Ce nombre est en augmentation de $20 \%$ par rapport à 2015. Un nouveau visage de nos ces personnes se dessine dans cette étude menée par Paris21Tv en partenariat avec la Société de Banque et d'Expansion. Cet article décline les changements observés en termes de profils des personnes et de leur représentation de la société française dans laquelle ils se sont installés.

\section{Un profil relativement stable}

Le profil de ces impatriés, salariés expatriés, détachés ou en mission locale - dirigeant d'entreprise - profession libérale de nationalité étrangère travaillant en France, ils ont en moyenne plus de 41 
ans et sont majoritairement de sexe masculin. II est est européen, Allemand ou Britannique majoritairement, et marié. II vit en famille le plus souvent et commence à délaisser Paris et sa région pour vivre en province avec une prédilection pour l'Occitanie. A la lecture de l'augmentation (+15 points) des impatriés mariés, gageons que ceux qui étaient célibataires ou vivaient maritalement sont passés depuis devant Monsieur le Maire, puisqu'ils sont désormais près de 7 sur 10 à être mariés.

L'impatrié est sur le territoire depuis plus de 5 ans et envisage de rester au moins 10 ans. II est cadre, salarié d'une grande entreprise, le plus souvent française. II est également en contrat local et bénéficie donc de moins d'avantage que ses homologues sous contrat "expatrié " ou " détaché " qui eux voient leur loyer et les frais de scolarité de leur enfant pris en charge.

Bien que $39 \%$ des répondants à l'enquête touchent plus de $65000 €$ par an, sa rémunération serait en baisse si l'on en juge par l'augmentation du segment qui concerne les revenus annuels inférieurs à 30 ooo $€$... une grande nouveauté depuis 2015. Mais ne serait-ce pas le créateur d'entreprise qui commence à arriver sur le territoire, qui ferait baisser cette rémunération?

\section{Des provenances européennes}

Ce qui change aujourd'hui, c'est leur provenance géographique. En 2015, nous avions constaté une baisse de près de $5 \%$ d'impatriés de l'Union Européenne contre une augmentation forte de $6 \%$ pour les pays hors UE, 4\% en Amérique du Nord. En 2016, les impatriés en provenance de l'Union Européenne sont de plus en plus nombreux (+8 points/2015). Les chiffres s'inversent pour les Impatriés hors UE.

Faut-il en déduire l'effet «brexit» notamment ? Oui mais pas seulement, quand on lit dans les colonnes du quotidien «Le Monde» du 22 mars 2017 : "L'Allemagne est devenue championne des implantations dans l'Hexagone, avec une progression de 35 \% du nombre de projets.». Quant aux Américains du Nord, traditionnellement premiers sur le podium des investisseurs en France ils sont, toujours bien présents (+ 2 points). L'État français depuis plusieurs années a souhaité rendre attractive l'impatriation de compétences techniques et managériales. Malgré cette volonté, la poursuite des évènements des attentats a contribué à faire baisser le flux touristiques mais également impactés l'arrivée d’Impatriés de certains pays.

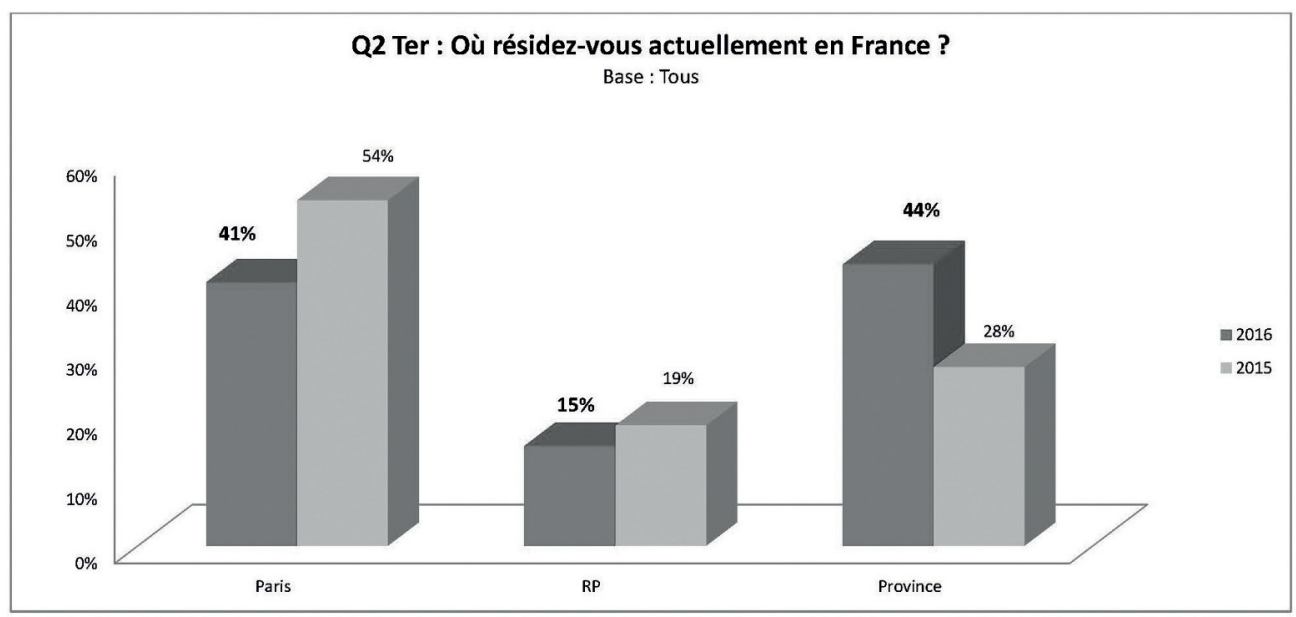




\section{REPÉRAGE}

Cette nouvelle carte géographique est cependant nuancée quant au choix du lieu de résidence. Alors qu'en 2015, plus de 1 impatrié sur 2 résidait à Paris, ils sont désormais plus nombreux à résider en province (44\%). Et même si ils sont tout de même majoritairement localisés à Paris et dans ses départements limitrophes, la mutation est à souligner. Une première depuis 3 ans que l'enquête existe, les impatriés font de plus en plus le choix de la province, ce qui influe, on le verra plus tard, dans l'enquête sur leur perception de la vie en France...Comme le révèle l'enquête, et comme on peut le constater dans différents médias, la Région Occitanie/Midi-Pyrénées est l'une des plus attractives du pays pour les investisseurs étrangers. Un attrait qui doit beaucoup à l'implantation de l'aéronautique et à la qualité de la R\&D, devenue une véritable culture territoriale.

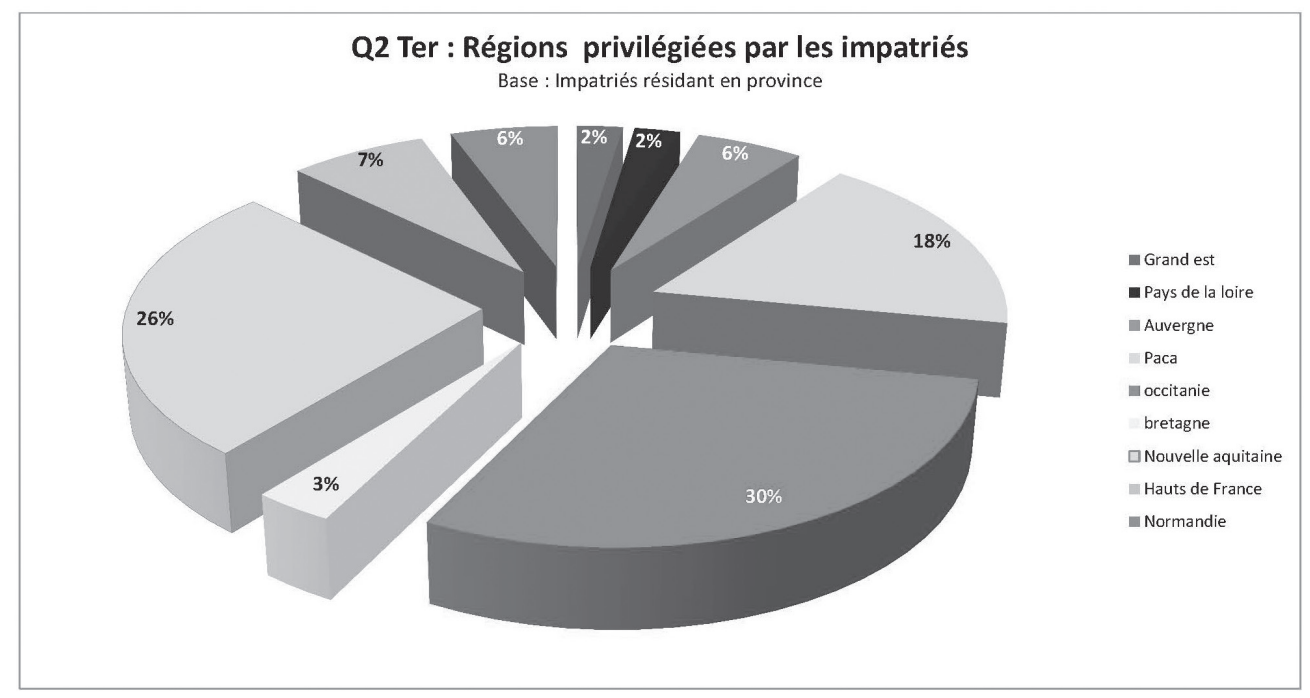

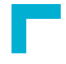

\section{Moins salarié et plus indépendant}

En terme d'insertion économique dans la société française, le constat de l'étude est que le nombre d'employés, qu'ils appartiennent à une structure étrangère (détaché ou expatrié) ou encore française (contrat local), est en nette baisse (-7 points) et ce en une seule année. Cette baisse profite aux indépendants qu'ils soient créateurs d'entreprise, commerçants ou encore professions libérales. Faut-il y voir un besoin d'indépendance chez les ex salariés, des résultantes d'offres proposées par les entreprises qu'elles soient françaises ou étrangères pour échapper aux charges patronales et à un code du travail qui pèsent sur elles ou encore de l'arrivée de nombreuses start up étrangères dans l'hexagone, suite par exemple à la mise en place du dispositif d'accueil des 70 lauréats du concours French Tech Ticket destiné aux entrepreneurs étrangers qui veulent monter ou développer leur start-up en France ? Dans tous les cas, on assiste à fin 2016 à un véritable tournant en termes de statut des impatriés et ce même si majoritairement les impatriés salariés en entreprise ont un statut de cadre. Cette mutation semble tendre vers l'auto entrepreneuriat et qui se vérifie dans l'hexagone, et ce même auprès de la population des impatriés. 


\section{Relations toujours difficiles avec les administrations}

En 2015, la complexité des relations avec l'administration notamment avec la Sécurité Sociale pour l'obtention de la carte vitale, s'était renforcée. Cette difficulté reste toujours majeure alors que la Préfecture, nouvelle "bête noire» arrivée sur le podium en 2015, tendrait à améliorer ses rapports avec les impatriés. Sinon les Français semblent ne pas être les seuls à rencontrer des problèmes avec l'administration fiscale, une administration dont les impatriés se plaignent de plus en plus (+7 points/2014) et ce malgré les «allègements fiscaux» qui leur sont octroyés notamment grâce aux mesures adoptées dans la dernière loi Sapin... La difficulté tient peut-être au fait que justement, il est compliqué pour eux de bénéficier de ces «remises»...

\section{Leur intégration en France}

En 2016 et pour la première fois depuis 3 ans, on constate un bouleversement dans les motivations des impatriés à venir s'installer en France. En effet, ni l'intérêt de la mission, ni la rémunération ou encore l'apprentissage de la langue et l'enrichissement culturel ne sont de réelles motivations pour ces «nouveaux» impatriés. C'est l'attrait de la vie en France qui est devenue une motivation majeure pour venir s'y installer. Ce bouleversement s'expliquerait par un changement des statuts de ces impatriés : plus d'indépendants, de créateurs d'entreprise qui privilégient la qualité de vie à l'intérêt d'une mission professionnelle ou encore d'une rémunération en baisse.

Comme en 2016, cette étude a fait l'objet d'une présentation à un auditoire averti de directeurs des ressources humaines et responsables de la mobilité professionnelles, au Palais de la Porte Dorée et en partenariat avec le Musée national de l'histoire de l'immigration, au cours du premier trimestre. Elle est consultable sur www.netsbe.fr. I

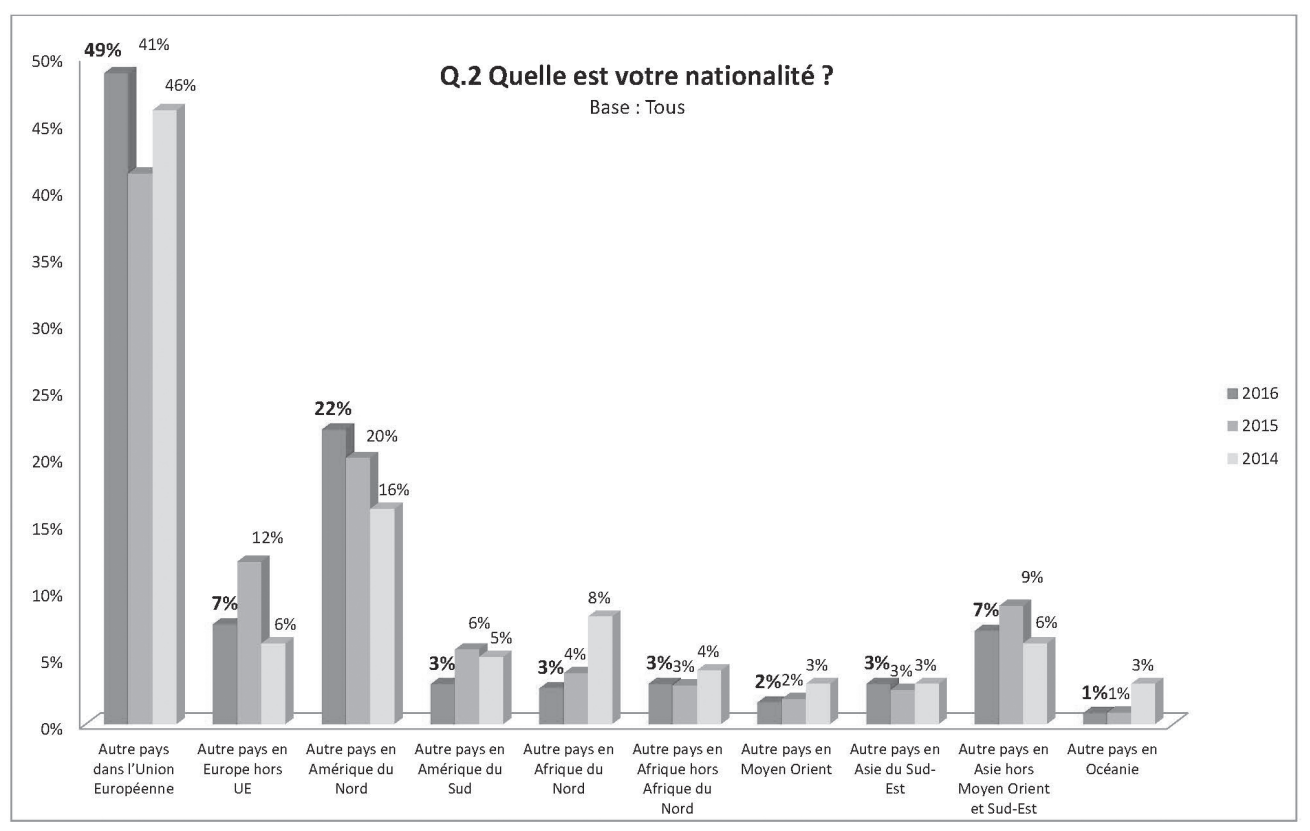

